\title{
An Ehrhart Series Formula For Reflexive Polytopes
}

\author{
Benjamin Braun \\ Department of Mathematics \\ Washington University, St. Louis, MO \\ math.wustl.edu/ bjbraun \\ bjbraun@math. wustl.edu
}

Submitted: Jul 12, 2006; Accepted: Sep 28, 2006; Published: Oct 5, 2006

Mathematics Subject Classifications: 52B20, 52C07

\begin{abstract}
It is well known that for $P$ and $Q$ lattice polytopes, the Ehrhart polynomial of $P \times Q$ satisfies $L_{P \times Q}(t)=L_{P}(t) L_{Q}(t)$. We show that there is a similar multiplicative relationship between the Ehrhart series for $P$, for $Q$, and for the free sum $P \oplus Q$ that holds when $P$ is reflexive and $Q$ contains 0 in its interior.
\end{abstract}

Let $P$ be a lattice polytope of dimension $d$, i.e. a convex polytope in $\mathbb{R}^{n}$ whose vertices are elements of $\mathbb{Z}^{n}$ and whose affine span has dimension $d$. A remarkable theorem due to E. Ehrhart, [4], asserts that for non-negative integers $t$ the number of lattice points in the $t^{\text {th }}$ dilate of $P$ is given by a degree $d$ polynomial in $t$ denoted by $L_{P}(t)$ and called the Ehrhart polynomial of $P$. We let

$$
\operatorname{Ehr}_{P}(x)=\sum_{t \geq 0} L_{P}(t) x^{t}=\frac{\sum_{j=0}^{d} h_{j}^{*} x^{j}}{(1-x)^{d+1}}
$$

denote the rational generating function for this polynomial (as in [7], chapter 4), called the Ehrhart series of $P$. See [3] for more information regarding Ehrhart theory. In this note we are concerned with a multiplicative decomposition for $\operatorname{Ehr}_{F}(x)$ when $F$ is a free sum of two lattice polytopes subject to some restrictions on the summands.

For two polytopes $P \subseteq \mathbb{R}^{d_{P}}$ and $Q \subseteq \mathbb{R}^{d_{Q}}$ of dimension $d_{P}$ and $d_{Q}$, define the free sum to be $P \oplus Q=\operatorname{conv}\left\{\left(0_{P} \times Q\right) \cup\left(P \times 0_{Q}\right)\right\} \subseteq \mathbb{R}^{d_{P}+d_{Q}}$. Let

$$
P^{\Delta}=\left\{x \in \mathbb{R}^{d_{P}}: x \cdot p \leq 1 \text { for all } p \in P\right\}
$$

denote the dual of $P$ and $P^{\circ}$ denote the interior of $P$. If $0 \in P^{\circ}$ and $0 \in Q^{\circ}$, then the free sum operation is dual to the product operation, i.e. $(P \times Q)^{\Delta}=\left(P^{\Delta}\right) \oplus\left(Q^{\Delta}\right)$, [5]. A basic example of this duality can be seen with the dual polytopes given by the $d$-dimensional crosspolytope and the $d$-dimensional cube, being the free sum of $d$ copies of the interval $[-1,1]$ and the product of $d$ copies of $[-1,1]$, respectively. 
From the perspective of Ehrhart theory, it is natural to ask which lattice polytopes have duals that are also lattice polytopes and hence have Ehrhart polynomials. A polytope with this property is called reflexive. Free sums of reflexive polytopes have recently played a crucial role in [2]. Reflexive polytopes have many rich properties, as seen in the following lemma.

Lemma 1 ([1] and [6]) $P$ is reflexive if and only if $P$ is a lattice polytope with $0 \in P^{\circ}$ that satisfies one of the following (equivalent) conditions:

i. $P^{\Delta}$ is a lattice polytope.

ii. $L_{P \circ}(t+1)=L_{P}(t)$ for all $t \in \mathbb{N}$, i.e. all lattice points in $\mathbb{R}^{d_{P}}$ sit on the boundary of some non-negative integral dilate of $P$.

iii. $h_{i}^{*}=h_{d_{P}-i}^{*}$ for all $i$, where $h_{i}^{*}$ is the $i^{\text {th }}$ coefficient in the numerator of the Ehrhart series for $P$.

For a product of polytopes, it is easy to see that $L_{P \times Q}(t)=L_{P}(t) L_{Q}(t)$. The following theorem indicates that Ehrhart polynomials also behave nicely for the free sum if a reflexive polytope is involved.

Theorem 1 If $P$ is a $d_{P}$-dimensional reflexive polytope in $\mathbb{R}^{d_{P}}$ and $Q$ is a $d_{Q}$-dimensional lattice polytope in $\mathbb{R}^{d_{Q}}$ with $0 \in Q^{\circ}$, then

$$
\operatorname{Ehr}_{P \oplus Q}(x)=(1-x) \operatorname{Ehr}_{P}(x) \operatorname{Ehr}_{Q}(x) .
$$

The key point in the following proof is that the $\mathbb{R}^{d_{P}}$ and $\mathbb{R}^{d_{Q}}$ components of lattice points in $t(P \oplus Q)$ cannot simultaneously be far from the origin. For what follows, consider vectors in $P$ and $Q$ as actually being in $P \oplus 0_{Q}$ and $0_{P} \oplus Q$, respectively.

Proof: Note that (1) is equivalent to

$$
L_{P \oplus Q}(t)=L_{Q}(t)+\sum_{k=1}^{t} L_{Q}(t-k)\left(L_{P}(k)-L_{P}(k-1)\right)
$$

for every $t \in \mathbb{N}$. This equivalence is seen by expanding the product on the right hand side of (1) as follows:

$$
\begin{aligned}
(1-x) \operatorname{Ehr}_{P}(x) \operatorname{Ehr}_{Q}(x) x i & =(1-x)\left(\sum_{r \geq 0} L_{Q}(r) x^{r}\right)\left(\sum_{s \geq 0} L_{P}(s) x^{s}\right) \\
& =\left(\sum_{r \geq 0} L_{Q}(r) x^{r}\right)\left(\sum_{s \geq 0} L_{P}(s) x^{s}-\sum_{s \geq 1} L_{P}(s-1) x^{s}\right) \\
& =\left(\sum_{r \geq 0} L_{Q}(r) x^{r}\right)\left(1+\sum_{s \geq 1}\left(L_{P}(s)-L_{P}(s-1)\right) x^{s}\right) \\
& =\sum_{t \geq 0}\left[L_{Q}(t)+\sum_{k=1}^{t} L_{Q}(t-k)\left(L_{P}(k)-L_{P}(k-1)\right)\right] x^{t} .
\end{aligned}
$$

We will therefore show that (2) holds for every $t \in \mathbb{N}$ and hence be done. 
Suppose first that $k$ is a real number between 0 and $t, t \in \mathbb{N}$. For $p \in k P, q \in(t-k) Q$, we have $\frac{p}{k} \in P, \frac{q}{t-k} \in Q$, and we see that

$$
\left(\frac{k}{t}\right)\left(\frac{t p}{k}\right)+\left(\frac{t-k}{t}\right)\left(\frac{t q}{t-k}\right)=\left[\begin{array}{c}
p \\
q
\end{array}\right] \in t P \oplus t Q=t(P \oplus Q) .
$$

Thus there is a copy of $k P \times(t-k) Q$ in $t(P \oplus Q)$ for any such $k$.

We will now show that $t(P \oplus Q)=\bigcup_{k} k P \times(t-k) Q$. Suppose $p \in \partial(k P)$ and $\left[\begin{array}{l}p \\ q\end{array}\right] \in t(P \oplus Q)$. We will show that $q$ cannot be outside $(t-k) Q$.

Note that $(t P)^{\Delta}=\frac{1}{t} P^{\Delta}$. So,

$$
(t(P \oplus Q))^{\Delta}=\frac{1}{t}\left(P^{\Delta}\right) \times \frac{1}{t}\left(Q^{\Delta}\right) .
$$

If $\frac{p}{k} \in \partial P$ then there exists some $p^{\Delta} \in P^{\Delta}$ such that $p^{\Delta} \cdot \frac{p}{k}=1$. Thus

$$
p \cdot \frac{1}{t} p^{\Delta}=\frac{k}{t}\left(p^{\Delta} \cdot \frac{p}{k}\right)=\frac{k}{t}
$$

If $\frac{q}{\alpha} \in \partial Q$, where $\alpha>(t-k)$, then similarly there exists $q^{\Delta} \in Q^{\Delta}$ such that

$$
q \cdot \frac{1}{t} q^{\Delta}=\frac{\alpha}{t}\left(q^{\Delta} \cdot \frac{q}{\alpha}\right)=\frac{\alpha}{t}>\frac{t-k}{t} .
$$

But, we know that

$$
(P \oplus Q)^{\Delta}=P^{\Delta} \times Q^{\Delta}
$$

and thus

$$
\frac{1}{t}\left[\begin{array}{l}
p^{\Delta} \\
q^{\Delta}
\end{array}\right] \in \frac{1}{t}\left(P^{\Delta} \times Q^{\Delta}\right)
$$

has a dot product with $\left[\begin{array}{l}p \\ q\end{array}\right] \in t(P \oplus Q)$ of greater than one, a contradiction.

Thus, every lattice point in $t(P \oplus Q)$ can be assigned uniquely to a lattice point in $t P$ by projection onto the $\mathbb{R}^{d_{P}}$ coordinate. Further, as $P$ is reflexive, all of the lattice points in $\mathbb{R}^{d_{P}}$ are contained in the boundary of $k P$ for some $k \in \mathbb{N}$. Therefore, the lattice points in $t(P \oplus Q)$ can be partitioned as

$$
\bigcup_{k \in\{0, \ldots, t\}}\left\{\left[\begin{array}{c}
p \\
q
\end{array}\right]: p \in \partial(k P), q \in(t-k) Q\right\} .
$$

It is immediate that $(2)$ counts the lattice points in $t(P \oplus Q)$ using this partition.

Note that the product on the right hand side of (1) corresponds to multiplying the numerators of $\operatorname{Ehr}_{P}(x)$ and $\operatorname{Ehr}_{Q}(x)$ and dividing by $(1-x)^{d_{P}+d_{Q}+1}$. So, just as the product polytope $P \times Q$ induces a product of Ehrhart polynomials, we see that the free sum induces a product of the numerators of the Ehrhart series of the summands. It is 
interesting that the Ehrhart polynomial and the Ehrhart series are "dual" to each other in this sense. It would also be interesting to find other examples of duality involving polynomials and their associated series where similar patterns arise.

A simple example shows that this theorem does not hold in general. Given $P=[-2,2]$, we see that

$$
\operatorname{Ehr}_{P}(x)=\frac{1+3 x}{(1-x)^{2}},
$$

while

$$
\operatorname{Ehr}_{P \oplus P}(x)=\frac{1+10 x+5 x^{2}}{(1-x)^{3}} .
$$

Thus, even for normal polytopes, this relationship does not always hold.

However, reflexivity is not necessary either. If $0 \in Q$, the pyramid of $Q$ is given by $[0,1] \oplus Q$. Though $[0,1]$ is not reflexive, it is well known that

$$
\operatorname{Ehr}_{[0,1] \oplus Q}(x)=(1-x) \operatorname{Ehr}_{[0,1]}(x) \operatorname{Ehr}_{Q}(x) .
$$

Despite not being reflexive, $[0,1]$ shares the property with reflexive polytopes that the lattice point in $t[0,1]-(t-1)[0,1]$ lies on the boundary of $t[0,1]$. Since $[0,1]$ is "half" of a reflexive polytope, the lattice points in $t([0,1] \oplus Q)$ are "filtered" uniquely by the lattice points in $t[0,1]$ and hence we get our result. This behavior can be generalized as follows:

Corollary 1 Suppose that $P$ and $Q$ are as in Theorem 1 and that $\left\{H_{i}\right\}_{i=1}^{k}$ and $\left\{K_{j}\right\}_{j=1}^{l}$ are halfspaces of the form $H_{i}=\left\{y \in \mathbb{R}^{d_{P}}: y \cdot a_{i} \geq 0\right\}$ for some $a_{i} \in \mathbb{R}^{d_{P}}$ and $K_{j}=$ $\left\{u \in \mathbb{R}^{d_{Q}}: u \cdot b_{j} \geq 0\right\}$ for some $b_{j} \in \mathbb{R}^{d_{Q}}$, respectively. Set $H=\cap H_{i}$ and $K=\cap K_{j}$. If $H \cap P$ and $K \cap Q$ are lattice polytopes, then

$$
\operatorname{Ehr}_{(H \cap P) \oplus(K \cap Q)}(x)=(1-x) \operatorname{Ehr}_{H \cap P}(x) \operatorname{Ehr}_{K \cap Q}(x) .
$$

Proof: We can extend $H_{i}$ and $K_{j}$ to halfspaces $\hat{H}_{i} \subseteq \mathbb{R}^{d_{P}+d_{Q}}$ and $\hat{K}_{j} \subseteq \mathbb{R}^{d_{P}+d_{Q}}$ by setting

$$
\hat{H}_{i}=\left\{z \in \mathbb{R}^{d_{P}+d_{Q}}:\left[\begin{array}{c}
a_{i} \\
0_{Q}
\end{array}\right] \cdot z \geq 0\right\}
$$

and

$$
\hat{K}_{j}=\left\{z \in \mathbb{R}^{d_{P}+d_{Q}}:\left[\begin{array}{c}
0_{P} \\
b_{i}
\end{array}\right] \cdot z \geq 0\right\} .
$$

We will first show that

$$
t((H \cap P) \oplus(K \cap Q))=\left(\cap_{i} \hat{H}_{i}\right) \cap\left(\cap_{j} \hat{K}_{j}\right) \cap t(P \oplus Q) .
$$

For the "C" containment, let $\left[\begin{array}{l}p \\ q\end{array}\right] \in t((H \cap P) \oplus(K \cap Q))$. Then

$$
\left[\begin{array}{l}
p \\
q
\end{array}\right]=\sum \alpha_{m} p_{m}+\sum \beta_{n} q_{n}
$$

where each $p_{m} \in t(H \cap P)$, each $q_{n} \in t(K \cap Q), \sum \alpha_{m}+\sum \beta_{n}=1$, and $\alpha_{m}, \beta_{n} \geq 0$. 
Thus, for all $i$,

$$
\left[\begin{array}{c}
a_{i} \\
0_{Q}
\end{array}\right] \cdot\left[\begin{array}{c}
p \\
q
\end{array}\right]=\left[\begin{array}{c}
a_{i} \\
0_{Q}
\end{array}\right] \cdot\left[\begin{array}{c}
\sum \alpha_{m} p_{m} \\
\sum \beta_{n} q_{n}
\end{array}\right] \geq 0
$$

and, for all $j$,

$$
\left[\begin{array}{c}
0_{P} \\
b_{j}
\end{array}\right] \cdot\left[\begin{array}{c}
p \\
q
\end{array}\right]=\left[\begin{array}{c}
0_{P} \\
b_{j}
\end{array}\right] \cdot\left[\begin{array}{l}
\sum \alpha_{m} p_{m} \\
\sum \beta_{n} q_{n}
\end{array}\right] \geq 0
$$

Hence we see that $\left[\begin{array}{c}p \\ q\end{array}\right] \in\left(\cap_{i} \hat{H}_{i}\right) \cap\left(\cap_{j} \hat{K}_{j}\right) \cap t(P \oplus Q)$.

For the " $\supseteq$ " containment, let $\left[\begin{array}{l}p \\ q\end{array}\right] \in\left(\cap_{i} \hat{H}_{i}\right) \cap\left(\cap_{j} \hat{K}_{j}\right) \cap t(P \oplus Q)$. We know that $p \cdot a_{i} \geq 0$ for all $i, q \cdot b_{j} \geq 0$ for all $j$ and (from the proof of Theorem 1) that $\left[\begin{array}{l}p \\ q\end{array}\right] \in k P \times(t-k) Q$ for some real number $0 \leq k \leq t$. Thus, $\frac{p}{k} \in H \cap P, \frac{q}{t-k} \in K \cap Q$, and

$$
\left(\frac{k}{t}\right)\left(\frac{t p}{k}\right)+\left(\frac{t-k}{t}\right)\left(\frac{t q}{t-k}\right)=\left[\begin{array}{c}
p \\
q
\end{array}\right] \in t((H \cap P) \oplus(K \cap Q)),
$$

hence we are done with (5).

Therefore, to count the lattice points in $t((H \cap P) \oplus(K \cap Q))$ we can count the lattice points in $\left(\cap_{i} \hat{H}_{i}\right) \cap\left(\cap_{j} \hat{K}_{j}\right) \cap t(P \oplus Q)$. This we can do by using our partition (3) from the proof of Theorem 1 and restricting our count to points satisfying the halfspace inequalities. That is exactly what is being recorded by

$$
\operatorname{Ehr}_{(H \cap P) \oplus(K \cap Q)}(x)=(1-x) \operatorname{Ehr}_{H \cap P}(x) \operatorname{Ehr}_{K \cap Q}(x) .
$$

\section{References}

[1] Victor V. Batyrev. Dual polyhedra and mirror symmetry for Calabi-Yau hypersurfaces in toric varieties. J. Algebraic Geom., 3(3):493-535, 1994.

[2] Matthias Beck and Serkan Hoşten. Cyclotomic polytopes and growth series of cyclotomic lattices. Math. Res. Let., 13(4):607-622, 2006.

[3] Matthias Beck and Sinai Robins. Computing the Continuous Discretely. Springer. To appear 2006, preprint at math.sfsu.edu/beck/ccd.html.

[4] Eugène Ehrhart. Sur les polyèdres rationnels homothétiques à $n$ dimensions. C. R. Acad. Sci. Paris, 254:616-618, 1962.

[5] Martin Henk, Jürgen Richter-Gebert, and Günter M. Ziegler. Basic properties of convex polytopes. In Handbook of discrete and computational geometry, CRC Press Ser. Discrete Math. Appl., pages 243-270. CRC, Boca Raton, FL, 1997.

[6] Takayuki Hibi. Dual polytopes of rational convex polytopes. Combinatorica, 12(2):237-240, 1992.

[7] Richard P. Stanley. Enumerative combinatorics. Vol. 1. Cambridge University Press, Cambridge, 1997. Corrected reprint of the 1986 original. 